\title{
Hacia una ciudad compacta: Cuenca y sus áreas residenciales
}

Towards a compact city: Cuenca and its residential areas

Por:

María Augusta Mera P.

María Elizabeth Santacruz A.

Universidad de Cuenca

Recibido: 15 de febrero 2012 Aceptado: 15 de Marzo 2012

\begin{abstract}
Resumen:
Los asentamientos residenciales en la ciudad de Cuenca se han ido conformando a la par de intervenciones urbanísticas realizadas con la intención de planificar un crecimiento de la ciudad, o por sucesos que han transcurrido a lo largo del tiempo.

En la actualidad, la urbe cuenta con un modelo de asentamiento en lo que corresponde a los usos de suelo que subyace tras la "Ordenanza que sanciona el Plan de Ordenamiento Territorial del cantón Cuenca: Determinaciones para el uso y ocupación del suelo urbano" emitida por el Municipio de Cuenca en 1998 y reformada en el 2003. Este modelo ha permitido a la población facilidades de servicios, comunicación y un entorno apto para el desenvolvimiento de las actividades cotidianas; sin embargo, luego de transcurrida una década de su vigencia, se ha creído pertinente evaluar y ajustar dicho modelo con la intención de propender hacia un asentamiento compacto.

Esta investigación se ha enfocado en buscar aquellos recursos que permitan mejorar las condiciones existentes en el modelo de usos de suelo actual a la vez que se ha planteado una serie de estrategias y recomendaciones para avanzar hacia una ciudad compacta.
\end{abstract}

Palabras clave: Áreas residenciales, ordenación teritorial, Cuenca, Ecuador

\section{Abstract:}

Residential settlements in the city of Cuenca have been forming at the same time as urban interventions carried out with the intention of planning a growth of the city, or by events that have passed over time.

At present, the city has a model of settlement in which corresponds to the uses of soil underlying after the "Ordinance that punishes the Territorial ordering Plan of the Cuenca canton: determinations for the use and occupation of urban soil" issued by the municipality of Cuenca in 1998 and renovated in 2003. This model has allowed the population with facilities services, communication and an environment suitable for the development of activities of daily living; However, after a decade of their validity, is believed relevant to evaluate and adjust this model with the intention of encourage towards a compact settlement.

This research has been focused on those resources that allow to improve the existing conditions in current soil use model, at the same time that has raised a series of strategies and recommendations to advance toward a compact city.

Keywords: Residential areas, territorial organization, Cuenca, Ecuador 


\section{Introducción:}

El espacio residencial debe entenderse como el resultado de un complejo entramado de decisiones ya que no es únicamente un espacio habitable, sino una localización, facilidad de comunicación y servicios, un entorno físico y valores sociales.

En este contexto, la función residencial de la ciudad de Cuenca tradicionalmente emplazada en su caso antiguo, ha sido desplazada específicamente en las últimas décadas hacia la periferia. El centro de ella conserva parte de sus edificaciones destinadas a la vivienda, además de otros usos entre los que se distinguen el comercio, los servicios y la gestión y administración. No obstante, este crecimiento expansivo residencial hacia la periferia no siempre ha tenido lugar de forma continua, ya que ha dejado espacios intersticiales, generando un despilfarro del recurso suelo y de infraestructura.

Para mitigar estos problemas, en el año 1998, entró en vigencia la “Ordenanza que sanciona Plan de Ordenamiento Territorial del cantón Cuenca: Determinaciones para el uso y ocupación del suelo urbano", siendo éste un gran esfuerzo por regular este aspecto básico de la ordenación urbanística, pues la Ordenanza no solamente se preocupó de asignar los usos de suelo buscando una adecuada combinación de actividades compatibles y complementarias con la vivienda, evitando la especialización funcional; sino que además reguló el comportamiento de tales actividades mediante una serie de normas técnicas que deben adoptar los proyectos arquitectónicos, las cuales han sido trascendentales para que la ciudad mejore su calidad ambiental. En el 2003, entró en vigencia la "Reforma, actualización, complementación y codificación de la ordenanza que sanciona el Plan de Ordenamiento Territorial del cantón Cuenca: Determinaciones para el uso y ocupación del suelo urbano" (Ordenanza 2003), la misma que mantiene vigente el modelo de asentamiento residencial que subyace tras esta normativa.
No obstante, luego de trascurrida más de una década de su vigencia, surgen justificadamente varias interrogantes: ¿con qué intensidad cuantitativa y cualitativa se han instalado en las áreas residenciales cuencanas los usos de suelo previstos en las referidas ordenanzas en calidad de complementarios y compatibles?, ¿cuál ha sido el comportamiento de los usos de suelo emplazados en esos territorios urbanos?, ¿cuáles son las opiniones y hasta posiciones de los ciudadanos respecto a los usos que se han emplazado en sus barrios en calidad de complementarios y compatibles?; incluso ha sido válido indagar si han sido suficientes las regulaciones de las actividades vinculadas a los usos complementarios y compatibles previstos en tales ordenanzas y si son suficientes los usos de suelo previstos en ellas, de cara a atender las demandas de las familias que residen en los barrios.

Por lo que se vuelve fundamental evaluar el modelo de asentamientos residenciales que se halla vigente, con el principal objetivo de reformular dicho modelo de uso de suelo a fin de que éste sirva para una adecuada ordenación urbanística, que permita preservar y mejorar las condiciones de vida y trabajo de la población, así como la calidad ambiental de los espacios residenciales, procurando un modelo de asentamiento compacto y sostenible.

Las experiencias de planificación como son los modelos de ciudad que se han planteado a lo largo del proceso de conformación de las ciudades latinoamericanas, específicamente a partir del modelo de ciudad funcional, con las propuestas de planificación de Le Corbusier, Josep Lluis Sert, Paul Lester Wiener, y otros; las propuestas del modelo de ciudad compacta de Salvador Rueda ${ }^{1}$, y su reflexión sobre la ciudad mediterránea y la ciudad dispersa han sido importantes criterios para la realización de la investigación. Las experiencias de planificación en América Latina como el Plan de Ordenamiento Territorial de Cali (2000), el Plan Equinoccio 21 (Quito, 2004), el Plan de Desarrollo Estratégico de Morrón (2005), entre otros documentos, han sido muy valiosos para entender las actuales tendencias de planificación en América Latina, concluyendo que estos procesos de planificación en su gran mayoría tienden hacia un modelo de ciudad que promueve la dispersión.

\section{Metodología de trabajo}

Para abordar el tema de la planificación en la ciudad de Cuenca fue necesario conocer los procesos que se ha desarrollado en la urbe, los cuales han sido producidos "a grandes saltos", considerando como una fuerte influencia para la expansión de la ciudad en la década de 1950, el anteproyecto del Plan Regulador para la ciudad de Cuenca $(1947)^{2}$, poste 


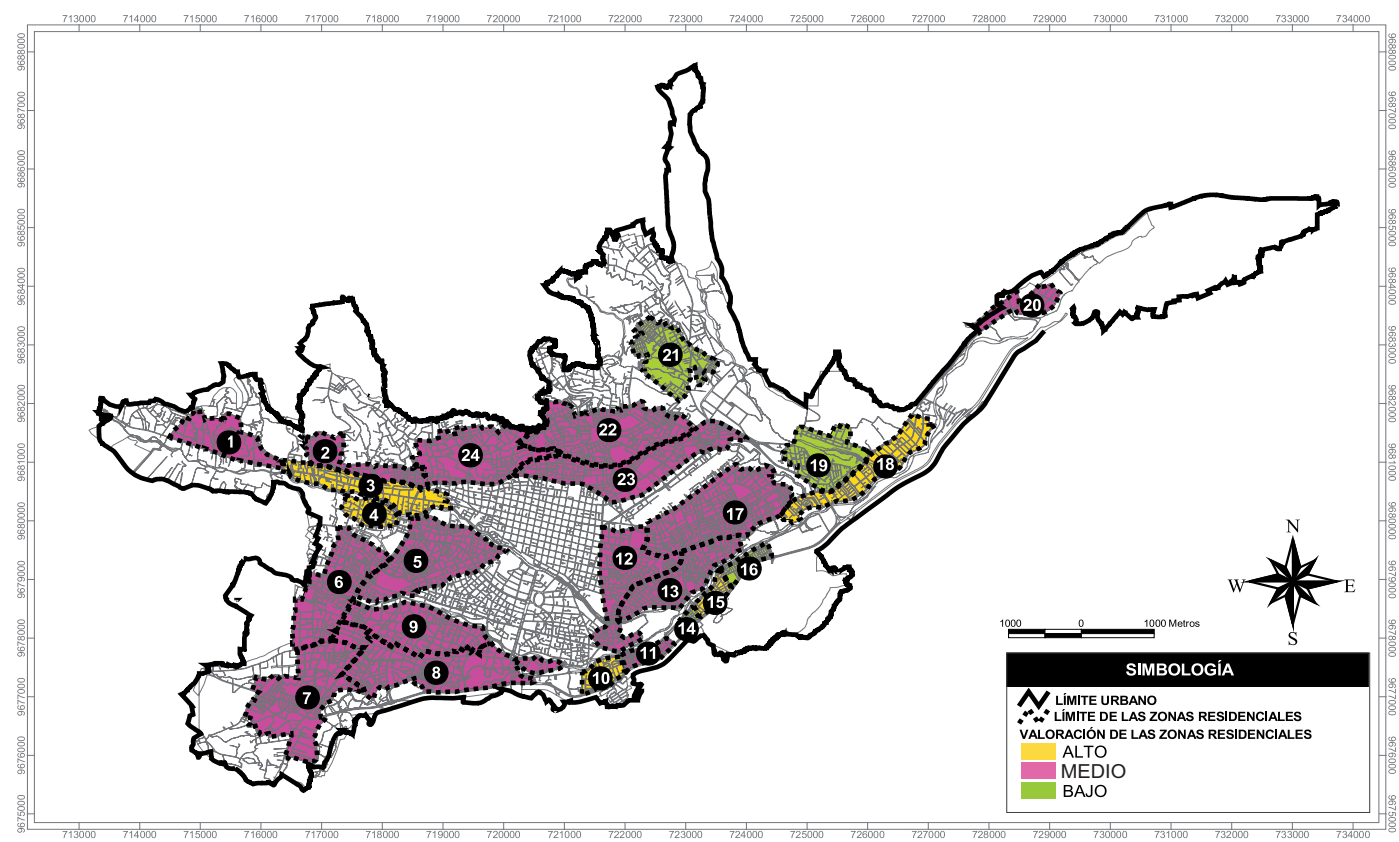

Gráfico № 1: Mapa de las zonas residenciales según su valoración socioeconómica Elaboración: Mera María Augusta y María Elizabeth Santacruz

Fuente: Mera María Augusta, María Elizabeth Santacruz, Fernando Pauta. “Modelo de usos de suelo para la gestión y administración municipal de las áreas residenciales de Cuenca”. Tesis previa a la obtención del título de Arquitectos. Cuenca, Ecuador: Universidad de Cuenca, Facultad de Arquitectura y Urbanismo, 2011, pág. 110.

riormente se realizó el Plan de Desarrollo Urbano del área metropolitana de Cuenca (1982), el mismo que mantuvo las directrices de planificación hasta la formulación del Plan de Ordenamiento Territorial del cantón Cuenca (1998), para ese entonces el perímetro urbano de la ciudad llegaba a las 6336 ha aproximadamente; en el año 2006, el área urbana era de $7227 \mathrm{ha}^{3}$, aumentando cada vez más su extensión debido a los procesos de crecimiento urbano que experimenta la ciudad. Sin embargo, este incremento no evidencia una urbe caótica y desordenada, aunque es indiscutible la necesidad de formular nuevas directrices de planificación que eviten las tendencias de ocupación de zonas periféricas y rurales con riesgo geológico o con limitaciones topográficas y que promuevan la utilización del suelo vacante de la ciudad.

Es importante mencionar que la demanda de vivienda en la ciudad no es homogénea, pues está condicionada por determinados factores como el nivel de ingresos económicos de las familias, la diferenciación de los segmentos sociales, el acceso al lugar de trabajo, la elección de la vivienda junto a un vecindario social semejante, los promotores inmobiliarios y los poderes públicos, el atractivo paisajístico, la posibilidad de contar con buenos servicios y la proximidad a las principales líneas de transporte son factores favorables a la existencia de áreas residenciales de nivel social superior; mientras que las áreas de paisaje monótono, la ausencia de servicios y la proximidad de la industria pesada o contaminante son factores que aparecen en la localización de las áreas de vivienda de estratos bajos, por lo que el desarrollo residencial en la ciudad de Cuenca no siempre presenta homogeneidad, ni en la calidad de la vivienda, ni en el nivel social de sus habitantes; de ahí la necesidad de determinar cuáles son los asentamientos dominantemente residenciales, con la finalidad de conocer sus características socioeconómicas y comprobar si existe o no una división social del espacio residencial, además de comprender cuál es tendencialmente el comportamiento de los usos de suelo en este territorio.

Para ello se identificaron 24 asentamientos, considerando ciertos criterios importantes para su demarcación como: excluir al Centro Histórico y El Ejido, ya que su dinámica es diferente -a pesar de que existe el uso residencial, estos territorios receptan mayoritariamente el comercio, los servicios, la gestión y administración-; no considerar las manzanas colindantes a equipamientos urbanos mayores (terminal terrestre, aeropuerto y el mercado del Arenal) debido al impacto en el uso del suelo, buscar rasgos homogéneos en cuanto a características socioeconómicas y edad de los asentamientos.

Las zonas residenciales estudiadas y valoradas en función de las características socioeconómicas se muestran en el Gráfico $\mathrm{N}^{\circ} 1$. Para la valoración se han considerado indicadores como la densidad poblacional, población alfabeta, nivel de instrucción de la población, población económicamente activa (PEA), PEA según grupos principales de 
ocupación y PEA según categoría ocupacional, con lo que se ha podido determinar que el $63 \%$ de los asentamientos residenciales analizados pertenece al estatus medio, el $21 \%$ al estatus alto y el $16 \%$ al estatus bajo. Sin embargo, vale la pena aclarar que el $8 \%$ del total de población registrada en las 24 zonas pertenece al estatus alto, el $11 \%$ al estatus bajo y el $81 \%$ al estatus medio, lo que demuestra que los grupos de ingresos altos son minoritarios en la ciudad de Cuenca y que el nivel de vida de la población en la urbe es bueno, ya que de igual manera el registro de población de estratos bajos es mucho menor que el de los estratos medios.

Con la finalidad de entender el comportamiento de los usos de suelo en el territorio residencial se han formulado indicadores como: el número de usos por cada cien viviendas, la densidad de usos, la diversidad de usos que relaciona el tipo y número de usos de suelo registrados, tomando como referencias estadísticas las medidas de tendencia central y de dispersión, la densidad de usos y densidad de población y el índice de Gini, el cual se ha aplicado con la finalidad de determinar la concentración o dispersión de usos de suelo en el territorio, que se encuentra representado en una muestra conformada por seis asentamientos, éstos se han seleccionado en función de la mayor aproximación a la mediana de las superficies de los mismos y en pares similares a fin de comparar los resultados entre zonas del mismo estatus y entre asentamientos de diferentes estatus.

El análisis realizado puede ser generalizado como una realidad de los usos de suelo en las áreas residenciales de la ciudad de Cuenca; sin embargo, es importante aclarar que los datos expuestos son valederos únicamente para esta urbe y que para otros casos podrían utilizarse solamente los métodos de comparación. En cuanto a los indicadores del número de usos por cada cien viviendas y a la densidad de usos de suelo, éstos son mayores en el comercio cotidiano de aprovisionamiento a la vivienda y en los servicios personales y afines a la vivienda, debido fundamentalmente a que los usos que engloba esta categoría son elementales para el desarrollo de las actividades diarias de la población, por lo que la demanda incrementa (Cuadro $\mathrm{N}^{\circ} .1$ ).

En lo que corresponde al equipamiento comunitario, las zonas residenciales de estatus socioeconómico bajo apuntan hacia un modelo sostenible de asentamiento, ya que es precisamente en estas zonas donde la dotación y uso de los equipamientos es mayor, por lo que su población en gran medida no se ve obligada a trasladarse a otros lugares de la ciudad en busca de este servicio, disminuyendo así los problemas de tránsito y transporte en la ciudad (Gráfico $\mathrm{N}^{\circ}$. 2).

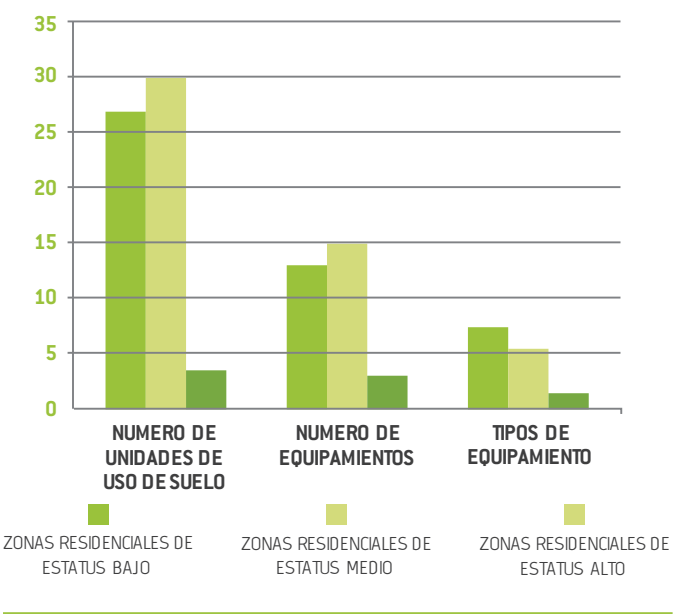

Gráfico № 2: Equipamiento comunitario registrado en las zonas residenciales tomadas como muestra

Elaboración: Mera María Augusta y María Elizabeth Santacruz

Fuente: Mera María Augusta, María Elizabeth Santacruz,, Fernando Pauta. "Modelo de usos de suelo para la gestión y administración municipal de las áreas residenciales de Cuenca". Tesis previa a la obtención del título de Arquitectos. Cuenca, Ecuador: Universidad de Cuenca, Facultad de Arquitectura y Urbanismo, 2011, pág. 278. 


\begin{tabular}{|c|c|c|c|c|c|c|c|c|}
\hline \multicolumn{9}{|c|}{ USOS COMPLEMENTARIOS } \\
\hline \multirow{2}{*}{$\begin{array}{c}\text { ZONAS } \\
\text { RESIDENCIALES }\end{array}$} & \multicolumn{4}{|c|}{$\begin{array}{c}\text { COMERCIO COTIDIANO DE } \\
\text { APROVISIONAMIENTO A LA VIVIENDA }\end{array}$} & \multicolumn{4}{|c|}{$\begin{array}{l}\text { SERVICIOS PERSONALES Y } \\
\text { AFINES A LA VIVIENDA }\end{array}$} \\
\hline & $\begin{array}{c}\text { NO. UNIDADES } \\
\text { DE USO } \\
\text { DE SUELO } \\
\end{array}$ & \begin{tabular}{|l} 
NO. DE USOS \\
POR CADA 100 \\
VIVIENDAS \\
\end{tabular} & $\begin{array}{l}\text { DENSIDAD } \\
\text { DE USOS } \\
\text { (usos/Ha) } \\
\end{array}$ & $\begin{array}{c}\begin{array}{c}\text { DIVERSIDAD DE } \\
\text { USOS }\end{array} \\
\text { NO. DE USOS } \\
\end{array}$ & $\begin{array}{c}\text { NO. UNIDADES } \\
\text { DE USO } \\
\text { DESUELO } \\
\end{array}$ & $\begin{array}{l}\text { NO. DE USOS } \\
\text { POR CADA } 100 \\
\text { VIVIENDAS } \\
\end{array}$ & $\begin{array}{l}\text { DENSIDAD } \\
\text { DEUSOS } \\
\text { (usos/Ha) } \\
\end{array}$ & \begin{tabular}{|c|} 
DIVERSIDAD DE \\
USOS \\
NO. DE USOS \\
\end{tabular} \\
\hline $\begin{array}{c}\text { ZONAS } \\
\text { RESIDENCIALES DE } \\
\text { ESTATUS BAJO }\end{array}$ & 130 & 7,29 & 1,28 & 9,5 & 67 & 3,94 & 0,66 & 9,5 \\
\hline $\begin{array}{c}\text { ZONAS } \\
\text { RESIDENCIALES DE } \\
\text { ESTATUS MEDIO }\end{array}$ & 140,5 & 5,92 & 0,91 & 9,5 & 86,5 & 3,64 & 0,56 & 9 \\
\hline $\begin{array}{c}\text { ZONAS } \\
\text { RESIDENCIALES DE } \\
\text { ESTATUS ALTO }\end{array}$ & 17,5 & 3,88 & 0,57 & 5,5 & 13 & 2,76 & 0,42 & 4,5 \\
\hline
\end{tabular}

Cuadro № 1: Indicadores de los usos de suelo complementarios en las zonas residenciales tomadas como muestra

Elaboración: Mera María Augusta y María Elizabeth Santacruz

Fuente: Mera María Augusta, SANTACRUZ María Elizabeth, Fernando Pauta. "Modelo de usos de suelo para la gestión y administración municipal de las áreas residenciales de Cuenca". Tesis previa a la obtención del título de Arquitectos. Cuenca, Ecuador: Universidad de Cuenca, Facultad de Arquitectura y Urbanismo, 2011, pág. 278.

\begin{tabular}{|c|c|c|c|c|c|c|c|c|c|c|}
\hline \multicolumn{11}{|c|}{ USOS COMPLEMENTARIOS } \\
\hline \multirow{2}{*}{$\begin{array}{c}\text { ZONAS } \\
\text { RESIDENCIALES }\end{array}$} & \multicolumn{3}{|c|}{ COMERCIO } & \multicolumn{3}{|c|}{ SERVICIOS GENERALES } & \multicolumn{4}{|c|}{$\begin{array}{l}\text { PRODUCCIÓN ARTESANAL Y MANUFACTURERA } \\
\text { DE BIENES COMPATIBLES CON LAVIVIENDA }\end{array}$} \\
\hline & $\begin{array}{c}\text { NO. UNID. } \\
\text { DEUSO } \\
\text { DESUELO } \\
\end{array}$ & $\begin{array}{c}\text { DENSIDAD } \\
\text { DE USOS } \\
\text { [usos/Ha) } \\
\end{array}$ & \begin{tabular}{|c|}
$\begin{array}{c}\text { DIVERSIDAD } \\
\text { DE USOS }\end{array}$ \\
NO. DEUSOS \\
\end{tabular} & $\begin{array}{c}\text { NO. UNID. } \\
\text { DEUSO } \\
\text { DESUELO } \\
\end{array}$ & $\begin{array}{c}\text { DENSIDAD } \\
\text { DEUSOS } \\
\text { (usos/Ha) } \\
\end{array}$ & \begin{tabular}{|c|} 
DIVERSIDAD \\
DEUSOS \\
NO. DEUSOS \\
\end{tabular} & $\begin{array}{c}\text { NO. UNID. } \\
\text { DEUSO } \\
\text { DESUELO } \\
\end{array}$ & \begin{tabular}{|c|} 
NO. DEUSOS \\
POR CADA 100 \\
VIVENDAS \\
\end{tabular} & $\begin{array}{c}\text { DENSIDAD } \\
\text { DE USOS } \\
\text { (usos/Ha) } \\
\end{array}$ & \begin{tabular}{|c|}
$\begin{array}{c}\text { DIVERSIDAD } \\
\text { DE USOS }\end{array}$ \\
NO. DE USOS \\
\end{tabular} \\
\hline $\begin{array}{l}\text { ZONAS } \\
\text { RESIDENCIALES DE } \\
\text { ESTATUS BAJO }\end{array}$ & 28,5 & 0,28 & 13 & 59 & 0,58 & 20 & 26,5 & 1,47 & 0,26 & 7 \\
\hline $\begin{array}{c}\text { ZONAS } \\
\text { RESIDENCIALES DE } \\
\text { ESTATUS MEDIO }\end{array}$ & 64 & 0,42 & 20,5 & 83,5 & 0,54 & 25,5 & 22,5 & 0,85 & 0,14 & 8 \\
\hline $\begin{array}{l}\text { ZONAS } \\
\text { RESIDENCIALES DE } \\
\text { ESTATUS ALTO }\end{array}$ & 6,5 & 0,25 & 6,5 & 16,5 & 0,51 & II & 4 & 0,86 & 0,13 & 3,5 \\
\hline
\end{tabular}

Cuadro № 2: Indicadores de los usos de suelo compatibles en las zonas residenciales tomadas como muestra Elaboración: Mera María Augusta y María Elizabeth Santacruz

Fuente: Mera María Augusta, María Elizabeth Santacruz,, Fernando Pauta. "Modelo de usos de suelo para la gestión y administración municipal de las áreas residenciales de Cuenca”. Tesis previa a la obtención del título de Arquitectos. Cuenca, Ecuador: Universidad de Cuenca, Facultad de Arquitectura y Urbanismo, 2011, pág. 279.

Los usos de servicios generales se presentan con mayor frecuencia y con mayor densidad que el comercio en las áreas residenciales, esto quiere decir que estas zonas se encuentran provistas de los bienes y servicios necesarios para el desenvolvimiento de actividades financieras, de comunicaciones, de recreación, de alimen- tación, etc., y que son usos que no se encuentran concentrados únicamente en el Centro Histórico. Si bien la producción de bienes tiene los valores más bajos en cuanto a densidad y número de usos por cada cien viviendas y se presentan con mayor intensidad en las zonas de estatus medio y bajo (Cuadro $\mathrm{N}^{\circ} 2$ ). 
Los usos de suelo catalogados como incompatibles con la vivienda se presentan con mayor intensidad en las zonas de estatus medio y bajo, su expresión en el territorio fundamentalmente son cerca de los ejes comerciales.

Al comparar las previsiones de la Ordenanza para las áreas residenciales y lo que sucede en el territorio se ha determinado que la estructuración de usos de suelo propuestos en el anexo 2 de la Ordenanza (2003) propicia a un modelo de ciudad compacta, el cual reúne una adecuada mezcla de usos, aportando así a una mayor eficiencia de recursos económicos y energéticos, este modelo posee mayor nivel de cumplimiento en los asentamientos de estatus socioeconómico medio y bajo, ya que es precisamente en estas zonas donde existe una mayor implantación de usos y una mayor diversidad, concibiendo a dichos sectores como lugares sustentables.

Sin embargo, es necesario realizar una revisión y una nueva codificación de los usos propuestos en el modelo de la Ordenanza (2003), ya que algunos usos han desaparecido, están absorbidos por otros o no deberían estar codificados para estos asentamientos; también es importante regular los usos complementarios y compatibles a la vivienda a fin de garantizar adecuadas condiciones de vida para la población residente, se cree que la asignación de usos de suelo a los ejes urbanos podría absorber ciertos usos que pueden ser incompatibles en las zonas residenciales. Es indispensable la formulación de políticas, estrategias e incentivos para la localización y relocalización de usos en el territorio de las áreas residenciales que permitan arribar a un modelo de ciudad sostenible.

En la propuesta del modelo de usos de suelo se contemplaron dos aspectos importantes, el primero es el "Diseño del modelo de usos de suelo para las áreas residenciales de Cuenca" y el segundo es la "Gestión del modelo" (Gráfico $\mathrm{N}^{\circ}$. 3).

La fase 1, que hace referencia al diseño del modelo, consta de componentes como: objetivos, asignación de usos de suelo y regulación de los usos de suelo previstos para el modelo.

Se definieron los principales objetivos para la concepción del modelo, los cuales estuvieron basados en los resultados del diagnóstico del comportamiento de los usos de suelo en las áreas residenciales de la ciudad de Cuenca. Para su planteamiento se consideró que el modelo de usos de suelo que prevé la Ordenanza 2003, actualmente vigente, apunta a buscar una ciudad sostenible y compacta que promueva una estructura social cohesionada, un territorio con cercanía a los servicios que propicie el encuentro de actividades, permitiendo el desarrollo de la vida en comunidad; por lo que se vuelve fundamental resguardar sus planteamientos. Según los resultados

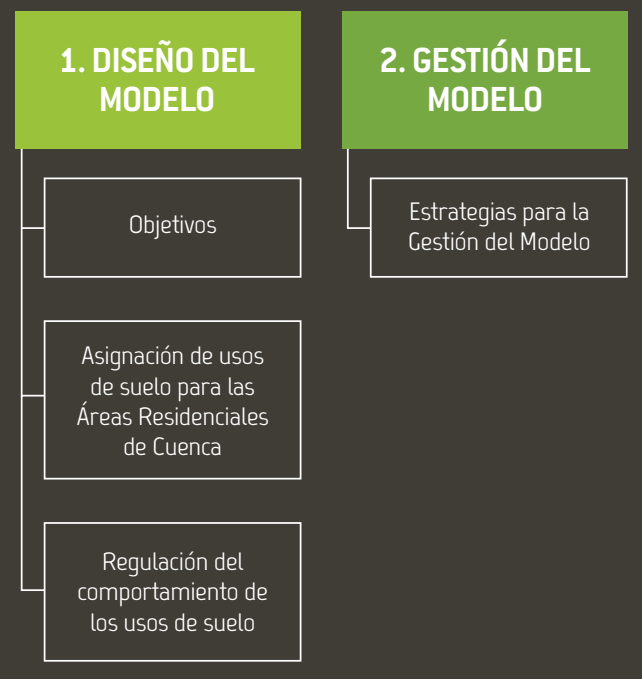

Gráfico № 3: Fases de la formulación del modelo de usos de suelo para las áreas residenciales de Cuenca

Elaboración: Mera María Augusta y María Elizabeth Santacruz

Fuente: Mera María Augusta, María Elizabeth Santacruz,, Fernando Pauta. "Modelo de usos de suelo para la gestión y administración municipal de las áreas residenciales de Cuenca". Tesis previa a la obtención del título de Arquitectos. Cuenca, Ecuador: Universidad de Cuenca, Facultad de Arquitectura y Urbanismo, 2011, pág. 327. 
obtenidos en el diagnóstico del comportamiento de usos de suelo en los asentamientos residenciales estudiados en calidad de muestra, observamos que se aproximan significativamente a este modelo que es motivo de expresión jurídica de la Ordenanza, en consecuencia la ciudad tiende a un crecimiento compacto. Por ello se creyó fundamental reforzar el modelo de ciudad compacta y sostenible, ya que mitiga los problemas de dispersión, segregación socio-espacial, tránsito, transporte, accesibilidad, despilfarro de recursos, al tiempo que promueve mejorar la calidad de vida de la población.

Con estas consideraciones se formularon objetivos como:

Avanzar hacia un modelo de uso de suelo para las áreas residenciales de la ciudad de Cuenca, que propicie un modelo de ciudad compacta, sostenible y eficaz, mediante la configuración de una red de asentamientos residenciales diversa en lo funcional e incluyente en lo social, que garantice el contacto, intercambio y comunicación entre personas y actividades, sin comprometer la calidad de vida de la población, esto con la intención de evitar las tendencias hacia la segregación socio-espacial de la población, frenar la dinámica de generación de la renta del suelo causa y efecto de la producción de "zonas exclusivas" que comienzan a aparecer en sectores como Río Sol, Cazhapata, Jardines del Río; entre otras urbanizaciones de la ciudad de Cuenca.

- Consolidar para Cuenca una red de asentamientos bien adaptados al aprovechamiento sostenible de los recursos locales, que cubran las necesidades y aspiraciones de la población cuencana, proveyendo a estos territorios de los bienes y servicios que demanda la vida cotidiana, para aliviar las centralidades urbanas y evitar el desplazamiento de la población a otros sectores de la ciudad como el Centro Histórico, sector del mercado El Arenal, El Ejido, etc., en busca de bienes y servicios cotidianos, coadyuvando a mitigar las demandas de transporte que se están generando en la ciudad y reduciendo la movilidad horizontal y el número de viajes diarios de la población, además de incentivar al aprovechamiento y ocupación del suelo urbano vacante, el cual actualmente asciende a un aproximado de $810 \mathrm{ha}^{4}$.

- Prevenir la generación de incompatibilidades de usos de suelo y la consecuente generación de impactos ambientales negativos, para garantizar el derecho de la población a vivir en un ambiente sano, ecológicamente equilibrado y libre de contaminación, permitiendo usos de suelo compatibles con la capacidad de la red vial existente en las áreas residenciales, que en la mayoría de los casos tiene una sección horizontal de ocho a diez metros de ancho, evitando de esta manera conflictos de tránsito y trasporte y resguardando la seguridad del peatón.

- Admitir usos de suelo relacionados con la agricultura urbana para contribuir a la seguridad alimentaria y promover la sostenibilidad de la ciudad mediante el incentivo a la utilización de lotes baldíos, áreas verdes, jardines, terrazas de edificios, bandas para plantar, etcétera.

Finalmente se creyó importante mantener en las áreas residenciales de la ciudad de Cuenca los usos de suelo tradicionales: comercio, servicio y producción.

En la asignación de usos de suelo para las áreas residenciales se consideró de vital importancia que éstos se encuentren establecidos de tal forma que permitan el desarrollo de un ambiente sano y ecológicamente equilibrado, ya que estas actividades tienen lugar en las zonas de residencia. Para ello se planteó una estructura de usos que afianza los objetivos trazados y una propuesta de codificación de los usos de suelo en los que se definieron los usos principales, complementarios, compatibles y prohibidos.

Como el principal uso de suelo es la vivienda, se creyó conveniente proponer que los usos complementarios sean todos aquellos tipos de usos de suelo de los cuales la población residente se sirve para el diario desenvolviendo de sus actividades. Para el efecto fueron considerados: el equipamiento comunitario de alcance barrial o parroquial, el equipamiento de apoyo a la producción, comercio cotidiano de productos de aprovisionamiento a la vivienda y los servicios personales y afines a la vivienda.

Los usos de suelo compatibles corresponden a aqueIlas actividades cuya coexistencia resulta admisible con la actividad principal y complementaria ya que sus impactos ambientales no afectan significativamente al desarrollo de las mismas. Los usos de suelo que se propuso en el modelo son: el comercio ocasional de productos de aprovisionamiento a la vivienda al por menor, comercio de repuestos y accesorios automotrices, comercio de maquinaria liviana y equipos en general y repuestos y accesorios, comercio de materiales de construcción y elementos accesorios, servicios financieros, servicios de transporte y comunicaciones, servicios de turismo y recreación, servicios de alimentación, servicios profesionales, servicios de seguridad, servicios industriales y producción artesanal y manufacturera de bienes compatible con la vivienda.

Se consideran como usos de suelo prohibidos en las áreas residenciales: los servicios industriales e industrias de mediano y bajo impacto (Tipo A), industrias de alto impacto (Tipo B); y todos aquellos que sean incompatibles con la vivienda.

En el caso de usos de suelo no previstos en el modelo, su aceptación queda condicionada a que éstos sean susceptibles de asimilarse a uno de los grupos de usos complementarios o compatibles. El uso deberá demandar para su emplazamiento espacios construidos, cubiertos y descubiertos y consumo de 
servicios básicos similares a los usos de suelo asignados, tener un horario de funcionamiento similar al de los establecimientos correspondientes a los usos de suelo previstos, no provocar impactos ambientales que interfieran con los usos de suelo previstos y que su funcionamiento no comprometa riesgos mayores a los que generan los usos de suelo previstos, por su naturaleza.

La regulación del comportamiento de los usos de suelo se encuentra conformada por una serie de normas técnicas que garantizan el desarrollo óptimo de las actividades, lo que evita la generación de impactos ambientales negativos.

Para normar el comportamiento de los usos de suelo asignados como complementarios y compatibles se propuso diferentes campos de regulación, mismos que se detallan a continuación:

- Código y nombre del uso de suelo, área de suelo: se refiere a la fijación de la superficie de suelo donde funcionan las actividades, en la cual se propone áreas mínimas y máximas, esta determinación está en función de espacios complementarios que necesitan los usos para funcionar.

- Área de construcción, localización: es la recomendación de una propuesta de localización más adecuada dentro de los asentamientos, en función del tipo de actividad que se realiza.

- Influentes: son los servicios públicos y combustibles necesarios para el adecuado funcionamiento de la actividad.

- Requisitos administrativos: son los permisos respectivos por parte de diferentes entidades públicas.

- Normas a observar: es el conjunto de normativas y reglamentos vigentes de diferentes entidades del país.

- Efluentes: se refiere al tratamiento que debe darse a los desechos que genera la actividad, o a las medidas a tomar para evitar la generación de impactos ambientales negativos.

- Horarios de funcionamiento y espacios complementarios: son todos aquellos espacios o dependencias adicionales con las que deben contar los locales para su funcionamiento.

Para la elaboración de las regulaciones y de las prácticas ambientales se han considerado las normativas ecuatorianas vigentes en materia de salud, sanidad, normas ambientales, construcción, etcétera.

En la fase 2, que hace referencia a la gestión del modelo, se apunta a formular un conjunto de estrategias que permitan alcanzar los objetivos y planteamientos propuestos para la relocalización, y regular el comportamiento de los usos de suelo.

Las estrategias para la relocalización de los usos de suelo describen diferentes aspectos como: la reforma de la Ordenanza actual, con la finalidad de realizar una actualización y recodificación de los usos de suelo, la creación de programas de relocalización de los equipamientos educativos, de salud y otros hacia las zonas residenciales, mejorando así la planificación con respecto al equipamiento público y privado; además de incentivar a la zonificación de la matrícula escolar en establecimientos educativos, se propone buscar alternativas de localización y nuevas ofertas para el pago de servicios básicos mediante la creación de ventanillas de pago en locales como tiendas de abarrotes, panaderías, papelerías, farmacias o cualquier actividad económica de la cual la población se sirve cotidianamente.

Para producir un crecimiento compacto y acelerar los procesos de consolidación urbanística de los barrios de la ciudad de Cuenca se propone reconsiderar el cobro del impuesto al solar no edificado, el cual debe realizarse en base al valor real del avalúo del terreno y fijar plazos para la edificación en lotes baldíos. Crear sistemas de centralidad barrial que recepte los usos que se han de desconcentrar del Centro Histórico de Cuenca ayudaría a evitar la dependencia de cierto tipo de usos con esta parte de la urbe, para ello es necesario la formulación de planes especiales de urbanismo, a fin de garantizar una óptima mezcla de usos de suelo y evitar la saturación de éstos en el territorio.

Además de lo mencionado, las estrategias de relocalización apuntan a crear canales de información y de venta de productos en los barrios residenciales, mediante la promoción comercio electrónico, la utilización de una red de internet gratuito para consulta de localización de bienes y servicios y la implementación del sistema de teletrabajo en instituciones públicas y privadas; apuntan también a estimular la creación de convenios entre los productores de bienes y servicios y gremios de artesanos, Cámara de Comercio, Cámara de la Pequeña Industria, con la finalidad de informar cuáles son los barrios de la ciudad donde existe una menor oferta de bienes y servicios para planificar conjuntamente en qué sectores puede incentivarse su localización.

Asimismo se cree importante destinar sitios específicos de la ciudad para la implantación de usos de suelo incompatibles con la vivienda e informar a la población sobre las sanciones que podrían acreditarse por el incumplimiento de la relocalización. 


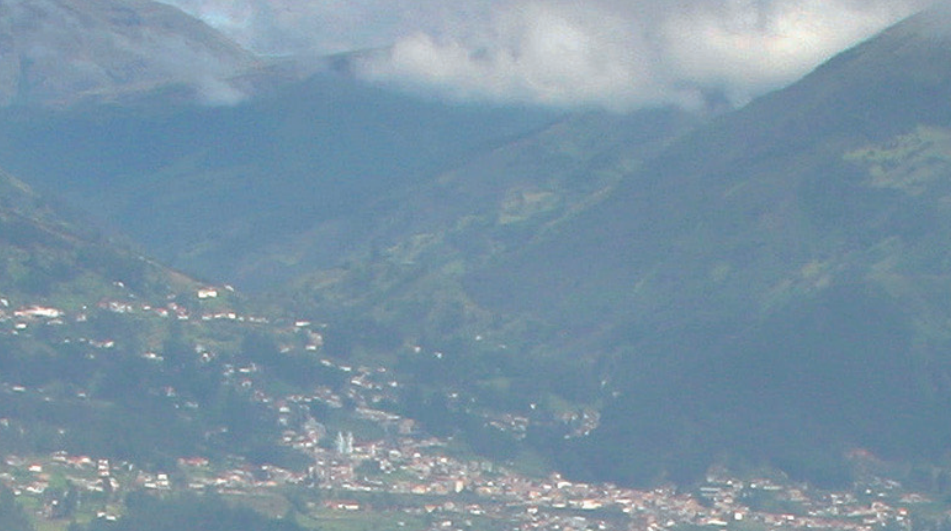

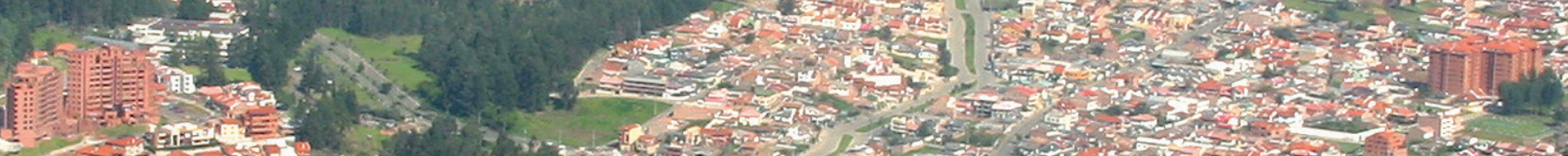

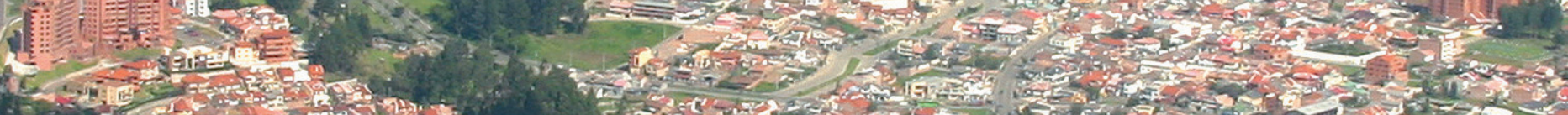

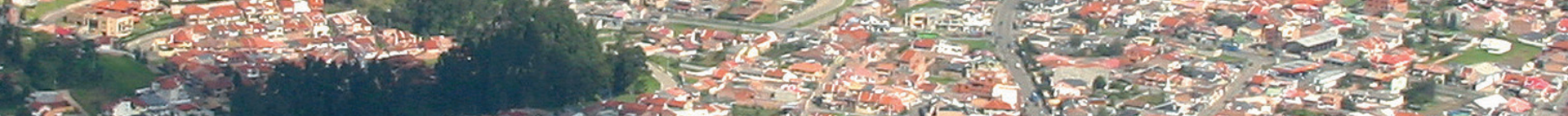

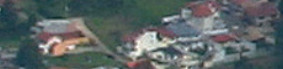
Het

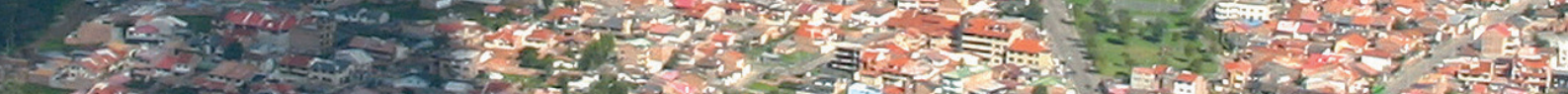

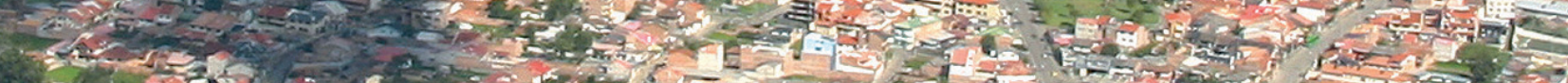

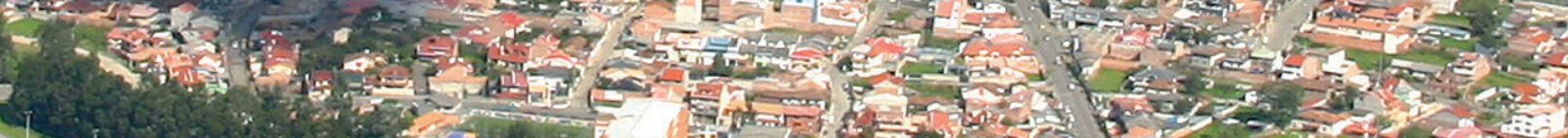

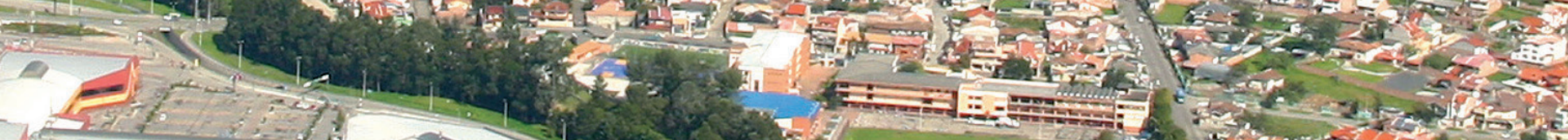

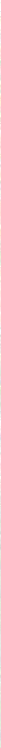


En lo referente a las estrategias para regular el comportamiento de los usos de suelo se creyó de vital importancia gestionar la implementación de las guías de prácticas ambientales con el objetivo de crear en los propietarios de los locales, una conciencia por la administración adecuada del agua, la energía eléctrica, la instalación de su local y el manejo de los efluentes mediante la implementación de locales municipales para brindar a la población la debida asesoría. De igual manera, se cree conveniente implantar un control municipal bajo una nueva concepción "más eficaz", orientando a los empleados municipales acerca de las normas y procedimientos a seguir para efectuar un óptimo control sobre el uso del suelo. Es necesario también crear herramientas apropiadas como la utilización de un SIG (Sistema de información geográfica) y una base de datos de uso de suelo, además de incentivar una cultura de respeto y orden por las normativas, así como la consolidación y la institucionalización, la participación ciudadana activa y responsable, promoviendo marcos regulatorios urbanísticos claros, concisos, prescriptivos y basados en incentivos y drásticas sanciones.

En la formulación de las estrategias de regulación es importante la creación de veedurías ciudadanas mediante las cuales se ejerza el derecho constitucional de controlar, de manera objetiva e imparcial, la administración y gestión de lo público, además de crear adecuados sistemas de información sobre las obligaciones y derechos que adquiere la población al promover actividades económicas en la ciudad y brindar información sobre los usos de suelo existentes en el barrio o en el territorio inmediato a los asentamientos residenciales mediante la implementación de cabinas de consulta ubicadas en puntos estratégicos de los asentamientos residenciales.

Finalmente, se propone establecer sanciones dirigidas al incumplimiento de las normas de regulación para cada actividad y la formulación de estrategias complementarias como son: incentivar los desplazamientos a pie o con medios no motorizados a través de un adecuado tratamiento de las aceras, de los espacios públicos y de las vías para transporte alternativo, y mejorar el servicio de transporte público, horarios de funcionamiento, etcétera.

\section{Conclusiones}

Se pudo constatar que las ciudades son, potencialmente, territorios con gran riqueza y diversidad económica, ambiental, política y cultural. Sin embargo, los modelos de desarrollo implementados en la mayoría de los países se caracterizan por establecer patrones de concentración de renta y poder que generan pobreza y exclusión, que conducen a la depredación del ambiente y aceleran los procesos migratorios y de urbanización, al tiempo que impulsan la segregación espacial y social, la extrema dependencia de la población de los sectores centrales de la ciudad y el uso indiscriminado del automóvil, situaciones que provocan congestión, contaminación ambiental y disminuyen la calidad de vida de los habitantes.

Para contrarrestar estos problemas se plantea como solución un modelo de ciudad compacta donde exista una sana mezcla de usos de suelo y funciones de la ciudad, el aumento de la compacidad da idea de una mayor proximidad, porque concentra en el espacio unidades de características diferentes, las hace más próximas y en consecuencia se reducen las distancias físicas. El tiempo para realizar las actividades se acorta y la energía dedicada a la movilidad será sustancialmente más pequeña, lo cual significa reducir la energía consumida por el sistema; motivo por el que resulta evidente que la ciudad compacta presenta mayores ventajas en comparación al modelo de ciudad funcional y dispersa. Por esta razón, son los fundamentos de la ciudad compacta los que se han utilizado para la propuesta del "Modelo de usos de suelo para las áreas residenciales de Cuenca", considerando además indispensable la formulación de estrategias particulares, y por tanto propias, para su gestión.

Finalmente, como recomendaciones para la aplicación del modelo propuesto es necesario permitir la localización de los usos de suelo previstos en todo el territorio de las zonas residenciales sin diferenciación de estratos sociales y al margen de la utilización de porcentajes para establecer intensidades de uso; aplicar el conjunto de estrategias para la localización y regulación del uso de suelo y todas las necesarias para avanzar hacia un asentamiento compacto y sostenible de ciudad; planificar las reservas de suelo para el equipamiento; propiciar un crecimiento compacto mediante la ocupación de los suelos vacantes y lotes sin edificar; establecer las regulaciones pertinentes para cada uno de los usos de suelo, especialmente aquellos que pueden tornarse conflictivos como por ejemplo los usos referentes a los servicios industriales; avanzar hacia una gestión de los usos de suelo que permita el control y la puesta en marcha de las estrategias y propuestas del modelo; fomentar la localización de usos relacionados con la producción artesanal y manufacturera de bienes compatibles con la vivienda para evitar la pérdida de este tipo de actividades. 


\section{Bibliografía}

${ }^{1}$ Rueda, Salvador. "Ciudad mediterránea, compacta y compleja. Una visión de futuro más sostenible". Barcelona: Ed. Ayuntamiento de Barcelona, 2002.

2 Jaramillo, Diego y Fernando Pauta. "La renta del suelo y la configuración del espacio residencial en Cuenca". Tesis previa a la obtención del título de Arquitectos. Cuenca, Ecuador: Universidad de Cuenca, Facultad de Arquitectura y Urbanismo, 1981.

Ceballos Leticia y Vilma Villavicencio. "El barrio como célula básica de ordenamiento territorial de áreas residenciales". Tesis previa a la obtención del título de Arquitectas. Cuenca, Ecuador: Universidad de Cuenca, Facultad de Arquitectura y Urbanismo, 1995.

${ }^{3}$ MERA María Augusta, María Elizabeth Santacruz, Fernando Pauta. "Modelo de usos de suelo para la gestión y administración municipal de las áreas residenciales de Cuenca". Tesis previa a la obtención del título de Arquitectos. Cuenca, Ecuador: Universidad de Cuenca, Facultad de Arquitectura y Urbanismo, 2011.

${ }^{4}$ Municipio de Cuenca, Plan de Ordenamiento Territorial del cantón Cuenca, Cuenca- Ecuador, 2003. 